\title{
Detection of TNT by a molecularly imprinted polymer film-based surface plasmon resonance sensor
}

\author{
BAO Han ${ }^{1}$, WEI TianXin ${ }^{1 *}$, LI XiuLi $^{1}$, ZHAO Zhe $^{2}$, CUI He${ }^{2} \&$ ZHANG Peng $^{3}$ \\ ${ }^{1}$ Key Laboratory of Cluster Science of Ministry of Education, Beijing Institute of Technology, Beijing 100081, China; \\ ${ }^{2}$ China Ship Research Development Academy, Beijing 100192, China; \\ ${ }^{3}$ Chinese Naval Academy of Armament, Beijing 100073, China
}

Received July 24, 2011; accepted November 17, 2011; published online April 23, 2012

\begin{abstract}
A surface plasmon resonance (SPR) sensor using a molecularly imprinted polymer (MIP) film as a recognition element for detection of 2,4,6-trinitrotoluene (TNT) was developed. The TNT-imprinted polymer film was prepared by thermo initiated polymerization on the bare Au surface of an SPR sensor chip. Template TNT molecules were quickly removed with an organic solution of acetonitrile/acetic acid $(9: 1, \mathrm{v} / \mathrm{v})$, causing a shift of $0.7^{\circ}$ in SPR angle. The limit of detection for TNT was demonstrated to be as low as $1 \times 10^{-8} \mathrm{~mol} / \mathrm{L}$. In the concentration range of $1 \times 10^{-8}-1 \times 10^{-5} \mathrm{~mol} / \mathrm{L}$, the change of SPR angle was linear with the negative logarithm of TNT concentration. Selectivity studies showed that the SPR sensor had no response to TNT analogues 2,4,5-trinitrotoluene and 1,3,5-trinitrohexahydro-1,3,5-triazine at a concentration of $1 \times 10^{-4} \mathrm{~mol} / \mathrm{L}$. The results suggest that the SPR sensor combined with a MIP film has the advantages of high sensitivity and selectivity, and long-term stability toward the analyte TNT.
\end{abstract}

TNT detection, surface plasmon resonance sensor, molecularly imprinted polymer film

Citation: Bao H, Wei T X, Li X L, et al. Detection of TNT by a molecularly imprinted polymer film-based surface plasmon resonance sensor. Chin Sci Bull, 2012, 57: 2102-2105, doi: 10.1007/s11434-012-5122-2

Because of the urgent needs of national security, human health and environmental protection, detection of nitroexplosives has attracted increasing attention in recent years [1]. 2,4,6-Trinitrotoluene (TNT) is a well-known explosive compound that has been widely used both in industry and military/terrorist activities. A variety of methods have been developed to determine the concentration of TNT including fluorescence [2-4], infrared [5], and luminescence spectroscopies [6], immunochemistry [7], and electrochemistry $[8,9]$. Of these, detection methods based on immunoassays are the most sensitive $[10,11]$, but they are time consuming and unstable, which makes them unsuitable for on-site detection. Thus, it is very important to develop a novel technology for detecting TNT rapidly and sensitively. One of the most promising approaches is surface plasmon resonance (SPR) sensors, which are small and portable for

*Corresponding author (email: txwei@bit.edu.cn) on-site detection. Recently, Kawaguchi et al. [12] reported a SPR sensor based on an antigen-antibody reaction for TNT determination. Riskin et al. [13] developed a bisanilinecross-linked $\mathrm{Au}$ nanoparticle matrix and achieved ultrasensitive detection of TNT using this SPR sensor.

SPR is a quantum optical-electrical phenomenon existing at the interface between a thin film of metal and a dielectric medium, and is extremely sensitive to changes in refractive index occurring within approximately a few hundred nanometers from the sensor surface [14]. Any change in the environment within this range will be transformed into an optical signal by an SPR sensor. Therefore, SPR sensors have become a central tool for characterizing and quantifying molecular interactions occurring at surfaces. Various types of recognition elements are available to allow SPR sensing platforms to be designed for specific detection of chemical and biological substances [15].

Molecularly imprinted polymers (MIPs) mimic the action 
of antibodies and enzymes to allow highly selective molecular recognition, and can be used as the recognition element in sensors [16,17]. MIPs have proven their potential in TNT detection because of their simplicity, reliability and low cost $[8,18,19]$. In the present work, we develop a novel SPR sensor platform for TNT detection using MIP film coated on an SPR chip. The particular recognition ability of the MIP template combined with SPR sensing characteristics might realize an ideal measurement system that is highly sensitive and selective, and relatively inexpensive for onsite detection of TNT and related compounds.

\section{Experimental}

\subsection{SPR measurements}

The SPR spectrometer used in this study (Figure 1) was homebuilt. From the He-Ne diode laser source $(632.8 \mathrm{~nm}$, $5 \mathrm{~mW}$ ), the light traveled through a chopper, polarizer and then into a sample cell. The sample cell consisted of a right-angled LaSFN9 prism in the Kretschmann configuration, which was coupled with a LaSFN9 slide $(2.5 \mathrm{~cm} \times 3.0$ $\mathrm{cm}$ ) using index-matching oil. The glass slide was used as a substrate for deposition of a layer of Au with a thickness of $47 \mathrm{~nm}$. A homebuilt flow cell $(0.8 \mathrm{~mL})$ was mounted over the slide using a rubber $o$-ring so that it tightly adjoined the gold surface. A peristaltic pump introduced sample liquid into the cell at a flow rate of $0.5 \mathrm{~mL} / \mathrm{min}$. The sample cell was placed on a circular stage. Reflected light from the sample cell was measured by a silicon photodiode detector.

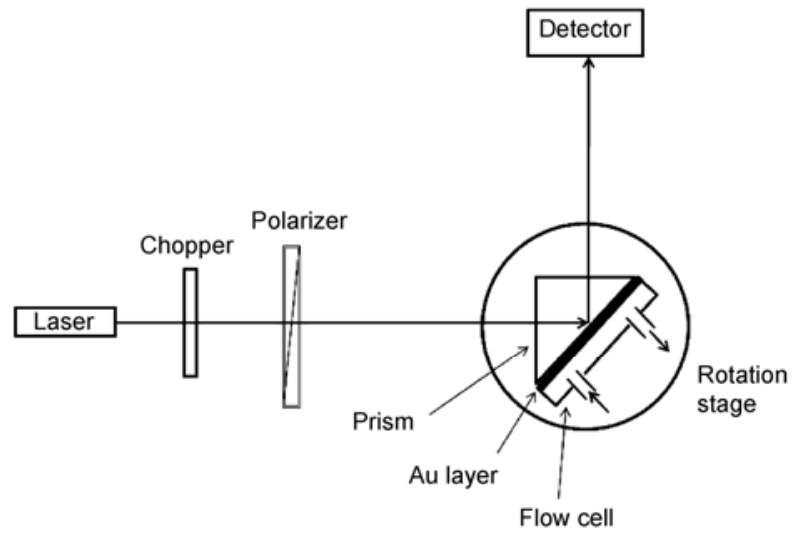

Figure 1 Schematic diagram of the homebuilt SPR sensor.

Its motion was controlled by a second rotation stage rotating at a rate of 2:1 (detector to prism). All data were collected and analyzed using in-house software.

\subsection{Preparation of TNT-MIP and non-imprinted polymer (NIP) films}

The MIP film was prepared by thermopolymerization on the bare gold surface of the SPR substrate as described in the ref.[20] with some modifications (as shown in Figure 2). First, the template molecule TNT $(0.025 \mathrm{~mol} / \mathrm{L})$ and the functional monomer acrylic acid (AA, $0.15 \mathrm{~mol} / \mathrm{L}$ ) were dissolved in acetonitrile $(3 \mathrm{~mL})$ and rested for $2 \mathrm{~h}$ to allow preorganization of template with the monomer. The crosslinker ethylene glycol dimethacrylate (EGDMA, $0.2 \mathrm{~mol} / \mathrm{L}$ )

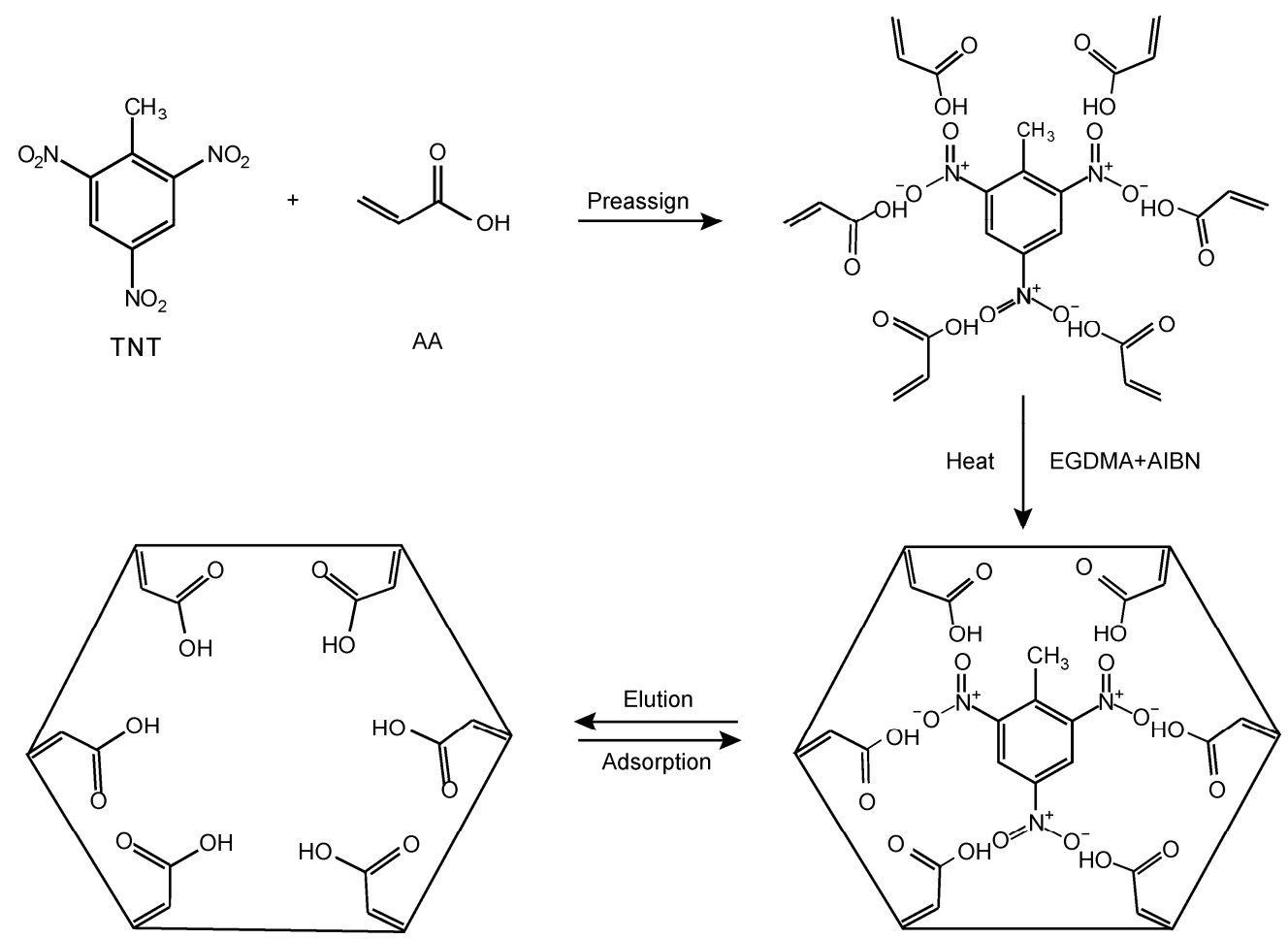

Figure 2 Preparation of a TNT-imprinted polymer film. 
and initiator 2,2'-azobis(2-methylpropionitrile) (AIBN, $0.012 \mathrm{~g} / \mathrm{mL}$ ) were then added to the pre-polymerization solution, followed by ultrasonication for $10 \mathrm{~min}$. The mixture was purged with nitrogen gas for $10 \mathrm{~min}$ and then added dropwise onto a glass slide coated with trimethylchlorosilane. The SPR substrate was immediately placed on the glass slide with the Au surface facing downwards and fastened tightly with clips. Parafilm was sandwiched between the two glass slides as a spacer. Polymerization was carried out at $60^{\circ} \mathrm{C}$ for $5 \mathrm{~h}$ under an atmosphere of nitrogen. A NIP film was synthesized as a reference under exactly the same conditions without template. The film coated on an SPR chip was washed with acetonitrile and ethanol several times and then dried under a stream of nitrogen gas.

\section{Results and discussion}

To create recognition cavities in the TNT-MIP film, removal of the template molecules was carried out following polymerization. Considering the non-covalent interaction between AA monomers and template TNT in the imprinted polymer, a mixture of acetonitrile and acetic acid was used to wash TNT from the template. Removal experiments were carried out by adding $5 \mathrm{~mL}$ of acetonitrile/acetic solution in volume ratios of $9: 1,8: 2$ and $7: 3$. Each solution was injected for $10 \mathrm{~min}$ and then the SPR response was measured in acetonitrile to assess the removal of TNT. Figure 3 shows SPR angular reflectivity spectra before and after rinsing with acetonitrile/acetic acid solution with a ratio of 9:1. A distinct shift of resonance angle from $68.9^{\circ}$ to $68.2^{\circ}$ resulting from a decrease in the refractive index of the film can be mainly attributed to removal of template TNT. The SPR angle did not change in subsequent washing experiments using an increased proportion of acetic acid, indicating that 9:1 is the optimum volume ratio allowing easy and thorough removal of TNT. In addition, the amount of crosslinker

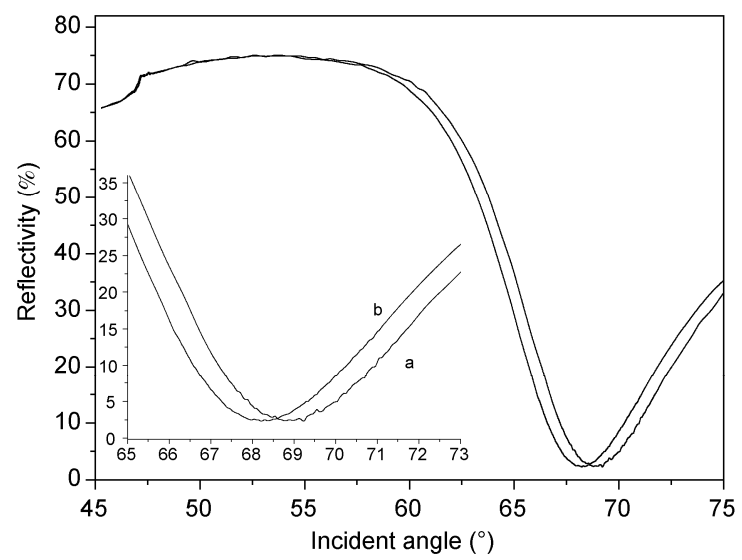

Figure 3 SPR angular reflectivity spectra of a TNT-MIP film in acetonitrile before (a) andafter (b) rinsing with acetonitrile/acetic acid (9:1, v/v). The inset shows an enlargement around the resonance angle. influenced the efficiency of template removal. An 8:1 molar ratio of crosslinker to template was optimal for this experiment. When the ratio was greater than $8: 1$, the resonance angle shifted less than $0.1^{\circ}$ after washing, which means that TNT molecules were not efficiently removed. When the ratio was less than $8: 1$, although the templates were easy to remove, the MIP films became unstable.

Adsorption experiments were used to further investigate the recognition ability of the MIP film. Solutions of TNT in acetonitrile with a range of concentrations from $10^{-8}$ to $10^{-5}$ $\mathrm{mol} / \mathrm{L}$ were successively injected into the flow cell at a flow rate of $0.5 \mathrm{~mL} / \mathrm{min}$. The injection of each TNT sample lasted for $900 \mathrm{~s}$ to reach equilibrium adsorption, and then the film was rinsed with background solvent (acetonitrile) for 300 s. As illustrated in Figure 4(a), a gradual change in the SPR dip during adsorption with increasing concentration of TNT indicated the capture of TNT molecules by imprinted sites. A plot of the change in resonance angle $(\Delta \theta)$ versus the negative logarithmic concentration of TNT $(-\lg [\mathrm{TNT}])$ exhibited a good linear response $\left(R^{2}=99.49 \%\right)$ in the
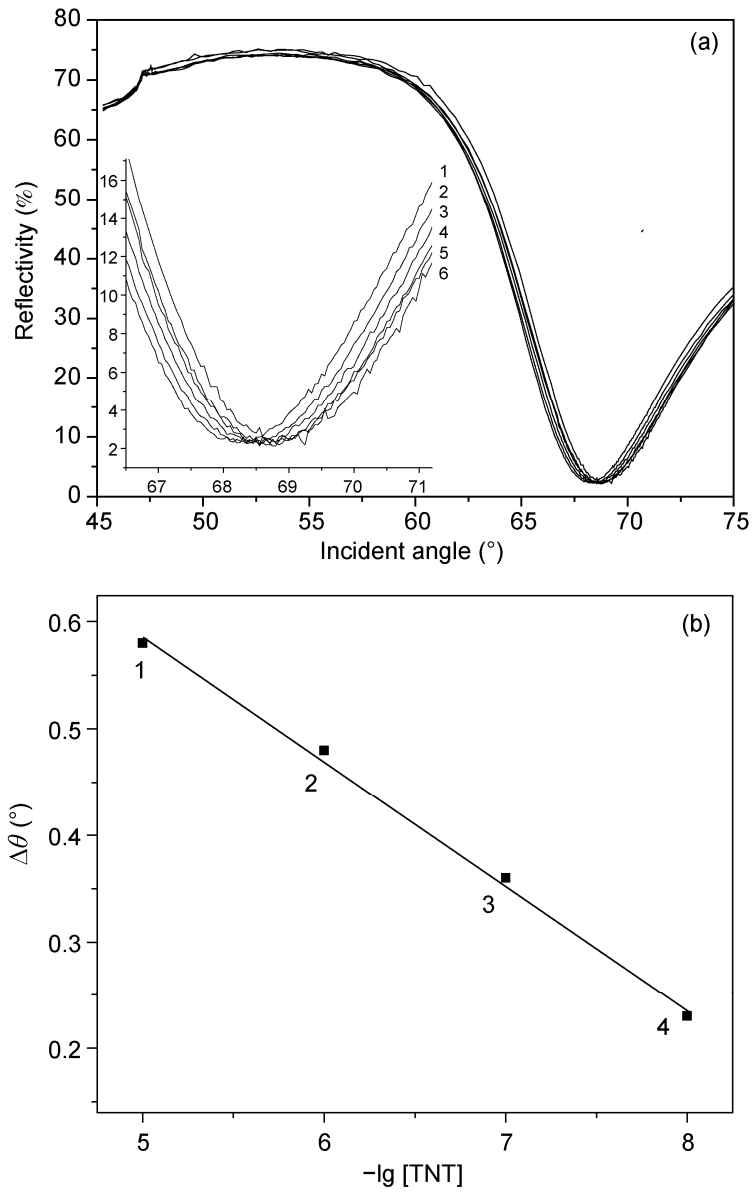

Figure 4 (a) SPR spectra of a TNT-MIP film after exposure to different concentrations of TNT. Curve 1, after rinsing; Curve 2-5, $1 \times 10^{-8}, 1 \times 10^{-7}$, $1 \times 10^{-6}$, and $1 \times 10^{-5} \mathrm{~mol} / \mathrm{L}$, respectively; and Curve 6 , before rinsing. Inset is an enlargement around the resonance angle. (b) Calibration curve of the change in SPR dip $(\Delta \theta)$ versus the negative logarithmic concentration of TNT, fitted with a linear function. 
concentration range of $10^{-8}$ to $10^{-5} \mathrm{~mol} / \mathrm{L}$, as can be seen in Figure 4(b). This suggests that the sensor chip readily adsorbs TNT and has a low limit of detection. As a control experiment, no noticeable change in SPR spectra were observed for a NIP film for TNT concentrations from $1 \times 10^{-8}$ to $1 \times 10^{-3} \mathrm{~mol} / \mathrm{L}$, which indicated that no specific or nonspecific binding of TNT occurred on the NIP. In addition, the MIP film exhibited good repeatability and stability after more than ten regeneration cycles.

The selectivity of the SPR sensor was evaluated by exposing it to structurally related analogues of TNT, 2,4,5trinitrotoluene (2,4,5-TNT) and 1,3,5-trinitrohexahydro-1,3, 5-triazine (RDX) (chemical structures are shown in Figure 5). As shown in Figure 6, there was no obvious change of the resonance angle (less than $0.1^{\circ}$ ) when these two samples dissolved in acetonitrile at a concentration of $10^{-4} \mathrm{~mol} / \mathrm{L}$ were exposed to the SPR chip. These results demonstrated that the TNT-imprinted films had very good selectivity for TNT molecules.

\section{Conclusions}

In summary, a novel SPR sensor containing a TNTimprinted polymer film as a recognition element was developed. The sensor demonstrated high sensitivity and selectivity for a non-labeling method that detects TNT. This work not only offers a new method for in situ monitoring of<smiles>Cc1c([N+](=O)[O-])cc([N+](=O)[O-])cc1[N+](=O)[O-]</smiles>

TNT<smiles>Cc1cc([N+](=O)[O-])c([N+](=O)[O-])cc1[N+](=O)[O-]</smiles>

2,4,5-TNT<smiles>O=[N+]([O-])N1CN([N+](=O)[O-])CN([N+](=O)[O-])C1</smiles>

RDX
Figure 5 Chemical structures of TNT and analogues.

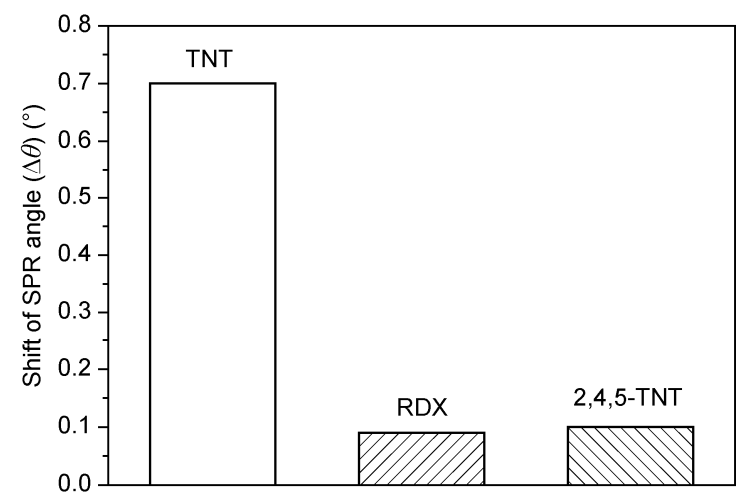

Figure 6 Change of SPR angle of a TNT-imprinted polymer film after adsorption with $10^{-4} \mathrm{~mol} / \mathrm{L}$ TNT, RDX and 2,4,5-TNT.
TNT but also has great potential for distinguishing TNT from other explosives.

This work was supported by the National Natural Science Foundation of China (20771015) and the Programme of Introducing Talents of Discipline to Universities (B07012).

1 Yinon J. Field detection and monitoring of explosives. Trends Anal Chem, 2002, 21: 292-301

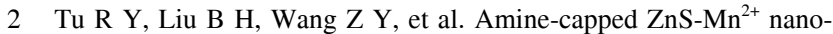
crystals for fluorescence detection of trace TNT explosive. Anal Chem, 2008, 80: 3458-3465

3 Yang J, Aschemeyer S, Martinez H P, et al. Hollow silica nanospheres containing a silafluorene-fluorene conjugated polymer for aqueous TNT and RDX detection. Chem Comm, 2010, 46: 6804-6806

4 Li H H, Lü F T, Zhang S J, et al. Preparation of monolayer-assembled fluorescent film and its sensing performances to hidden nitroaromatic explosives. Chin Sci Bull, 2008, 53: 1644-1650

5 Pushkarsky M B, Dunayevskiy I G, Prasanna M, et al. High-sensitivity detection of TNT. Proc Natl Acad Sci USA, 2006, 103: 19630-19634

6 Sohn H, Sailor M J, Magde D, et al. Detection of nitroaromatic explosives based on photoluminescent polymers containing metalloles. J Am Chem Soc, 2003, 125: 3821

7 Altstein M, Bronshtein A, Glattstein B, et al. Immunochemical approaches for purification and detection of TNT traces by antibodies entrapped in a sol-gel matrix. Anal Chem, 2001, 73: 2461-2467

8 Riskin M, Tel-Vered R, Bourenko T, et al. Imprinting of molecular recognition sites through electropolymerization of functionalized $\mathrm{Au}$ nanoparticles: Development of an electrochemical TNT sensor based on $\pi$-donor-acceptor interactions. J Am Chem Soc, 2008, 130: 97269733

9 Hrapovic S, Majid E, Liu Y, et al. Metallic nanoparticle-carbon nanotube composites for electrochemical determination of explosive nitroaromatic compounds. Anal Chem, 2006, 78: 5504-5512

10 Wilson R, Clavering C, Hutchinson A. Electrochemiluminescence enzyme immunoassay for TNT. Analyst, 2003, 128: 480-485

11 Green T M, Charles P T, Anderson G P. Detection of 2,4,6-trinitrotoluene in seawater using a reversed-displacement immunosensor. Anal Biochem, 2002, 310: 36-41

12 Kawaguchi T, Shankaran D R, Kim S J, et al. Fabrication of a novel immunosensor using functionalized self-assembled monolayer for trace level detection of TNT by surface plasmon resonance. Talanta, 2007, 72: 554-560

13 Riskin M, Tel-Vered R, Lioubashevski O, et al. Ultrasensitive surface plasmon resonance detection of trinitrotoluene by a bis-aniline-crosslinked $\mathrm{Au}$ nanoparticles composite. J Am Chem Soc, 2009, 131: 7368-7378

14 Homola J, Yee S S, Gauglitz G. Surface plasmon resonance sensors: Review. Sens Actuator B, 1999, 54: 3-15

15 Homola J. Surface plasmon resonance sensors for detection of chemical and biological species. Chem Rev, 2008, 108: 462-493

16 Sellergren B. Molecularly Imprinted Polymers: Man-Made Mimics of Antibodies and Their Applications in Analytical Chemistry. Amsterdam: Elsevier Publisher, 2001

17 Haupt B, Mosbach K. Molecularly imprinted polymers and their use in biomimetic sensors. Chem Rev, 2000, 100: 2495-2504

18 Xie C G, Zhang Z P, Wang D P, et al. Surface molecular self-assembly strategy for TNT imprinting of polymer nanowire/nanotube arrays. Anal Chem, 2006, 78: 8339-8346

19 Xie C G, Liu B H, Wang Z Y, et al. Molecular imprinting at walls of silica nanotubes for TNT recognition. Anal Chem, 2008, 80: 437-443

20 Wei Q Q, Wei T X, Wu X G. Ultrasensitive and highly selective detection of testosterone using a surface plasmon resonance sensor combined with molecularly imprinted films. Chem Lett, 2011, 40: 132-133

Open Access This article is distributed under the terms of the Creative Commons Attribution License which permits any use, distribution, and reproduction in any medium, provided the original author(s) and source are credited. 